\title{
Appendiceal pus in a hernia sac simulating strangulated femoral hernia: a case report
}

This article was published in the following Dove Press journal:

International Journal of General Medicine

22 March 20II

Number of times this article has been viewed

\author{
Tien-Fa Hsiao \\ Yenn-Hwei Chou \\ Department of Surgery, \\ Shin Kong Wu Ho-Su Memorial \\ Hospital, Taipei, Taiwan
}

Correspondence: Tien-Fa Hsiao Department of Surgery, Shin Kong Wu Ho-Su Memorial Hospital, No. 95, Wen Chang Road, Shih Lin District, Taipei III, Taiwan

Tel +8862283322I I ext 2086

Fax +8862 28389404

Email m002486@ms.skh.org.tw

\begin{abstract}
Acute appendicitis involving the hernia sac is infrequent but well-documented in medical literature. In most instances, it occurs within the right inguinal (Amyand's hernia) or right femoral hernia (de Garengeot hernia). The diagnosis is always mistaken for incarcerated groin hernia. During surgery, the appendix itself, either perforated or strangulated, is most commonly encountered within the hernia sac. In very rare occasions, only appendiceal pus is found in the hernia sac. In this paper, we report the case of a 90-year-old woman with acute appendicitis and a tender mass in the right groin. Typical findings of acute appendicitis by computed tomography (CT) and incarcerated femoral hernia with groin cellulitis misled us into preoperative diagnosis of strangulated femoral hernia. Acute phlegmonous inflammation of the incarcerated femoral hernia sac containing pus only and acute suppurative appendicitis were found intraoperatively. This case presents a rare complication of acute appendicitis and the first report of CT-documented appendiceal pus-contained femoral hernia. Knowledge of this rare condition is helpful in establishing preoperative diagnosis and patient management decisions.
\end{abstract}

Keywords: hernia sac, acute appendicitis, pus-containing femoral hernia

\section{Introduction}

Pus-contained femoral hernia sac is a very rare complication of acute appendicitis. It is most frequently presented with a mistaken diagnosis of a strangulated femoral hernia. Most reported cases have documented the development of significant complications, since it is difficult to diagnose this rare entity preoperatively. We present a case of appendiceal pus-containing femoral hernia. The physical finding simulates strangulated femoral hernia. Accurate preoperative diagnosis was made by computed tomography (CT). The literature is reviewed and the radiologic findings and management of this rare entity are discussed.

\section{Case report}

A 90-year-old woman was referred to our hospital for acute appendicitis complicated with right incarcerated groin hernia. She initially presented to a local hospital with right lower quadrant abdominal pain for one day. Blood investigations were unremarkable. The abdomen and pelvic CT with intravenous and oral contrast showed swelling of the appendix with perifocal fat stranding and cecal wall thickening (Figure 1A). The presumed diagnosis was acute appendicitis. As surgery was declined by her relatives, parenteral antibiotics were administered. There was initial improvement, however, a painful and irreducible lump in the right groin developed three days thereafter. On referral, a right incarcerated femoral hernia with local signs of redness and tenderness 

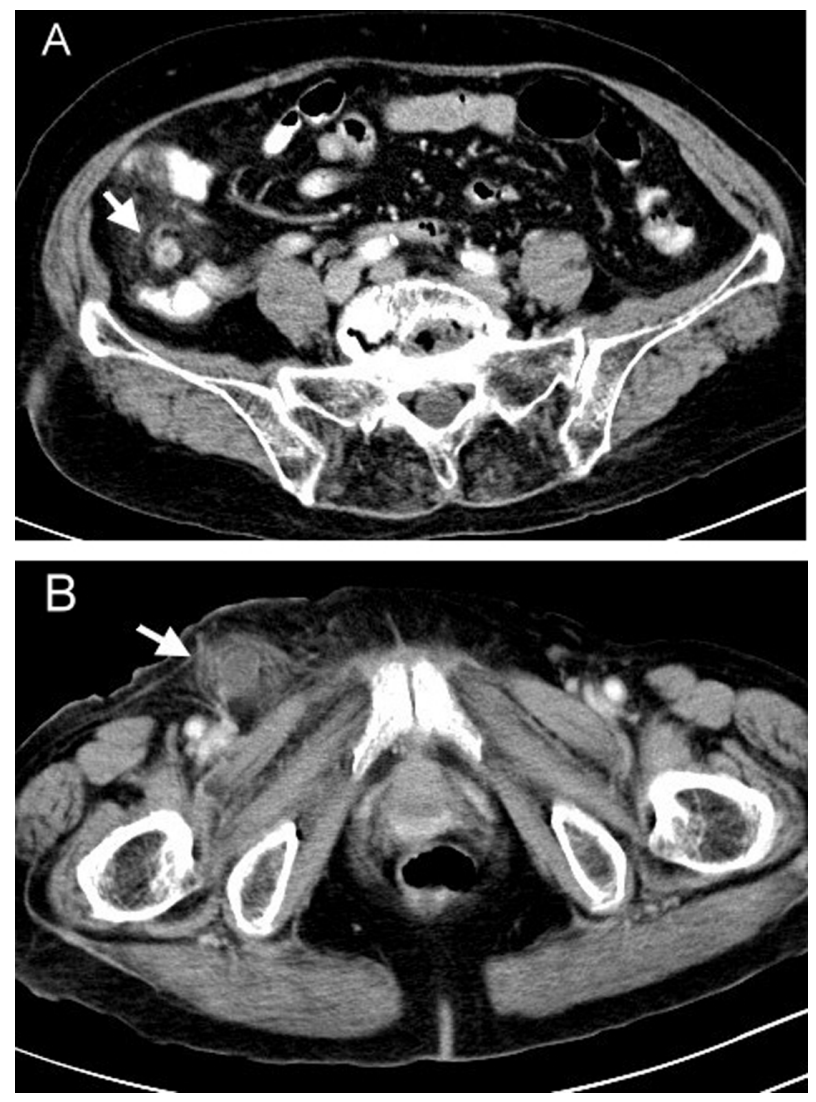

Figure I Abdomen CT with intravenous and oral constrast demonstrating swelling of appendix, thickened wall of cecum and perifocal fat stranding (A). The fluidcontained hernia sac was seen lateral and inferior to the pubic tubercle, with circumferential fat stranding (B)

was noted. Lower abdominal tenderness was also elicited. A review of a previously performed $\mathrm{CT}$ revealed the finding of a fluid-contained mass with stranding of surrounding fat in the right groin lateral and inferior to the pubic tubercle. No evidence of intestinal obstruction was found. Neither the appendix nor cecum was in close proximity to the mass (Figure 1B). Laparotomy was performed via lower midline incision. The appendix was inflamed and severely adherent to the cecum and terminal ileum but there was neither perforation nor abscess. The appendectomy was performed. The femoral hernia neck was constricted, and there was no herniation of the abdominal viscera (Figure 2A). A separate groin incision was made, and phlegmonous inflammation of the distended femoral hernia sac was found (Figure 2B). Dissection was carried out to free the entire circumference of the hernia sac and the neck. The hernia was reduced into the peritoneal cavity. The hernia sac was excised, and the neck was closed with suture-ligation. McVay hernioplasty was performed. On opening, the sole content of the hernia sac was pus. Bacterial culture grew Escherichia coli. Histology
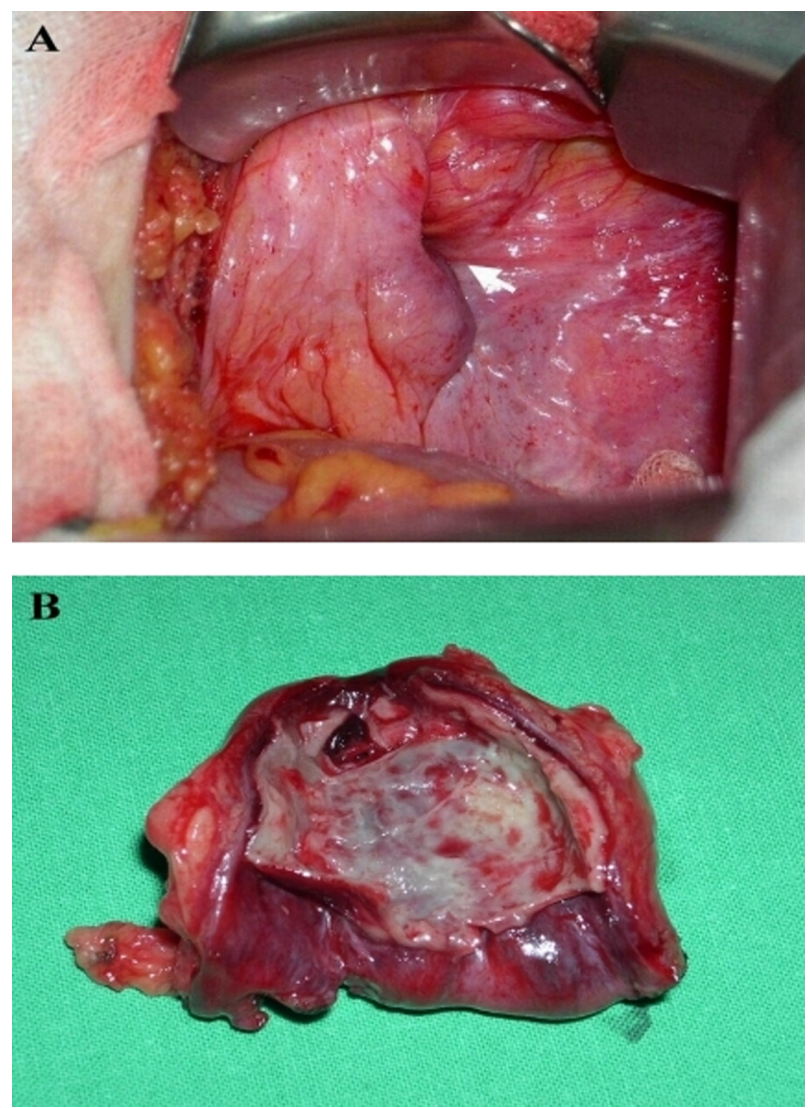

Figure 2 Intraoperative findings showed contricted hernia neck of the femoral hernia without herniation of abdominal viscera $(\mathbf{A})$, and acute phlegmonous inflammation of the incarcerated hernia sac (B).

revealed an infected hernia sac. The patient's postoperative recovery was uneventful.

\section{Discussion}

The reported incidence of appendicitis within a groin hernia is $0.13 \%$ of all cases of acute appendicitis. ${ }^{1}$ It most frequently occurs in the right inguinal and right femoral hernias. Rene Jacques Croissant de Garengeot, in 1731, first reported a case of acute appendicitis within a femoral hernia. ${ }^{2}$ Since then, there have been only scattered case reports of appendicitis within femoral hernias. The clinical presentation usually suggests an incarcerated or strangulated femoral hernia, and acute appendicitis is incidentally found at operation. With the increased use of CT for evaluating patient with atypical presentation of incarcerated hernia and lower abdomen peritonitis, there have been occasional reports in which the diagnosis of hernial appendicitis is made preoperatively. The typical CT features include a tubular structure within the hernia sac with surrounding fat stranding and low position of the cecum or close proximity to the hernia sac..$^{3-5}$ 
This prospective CT diagnosis may be helpful in establishing appropriate treatment strategy.

Our case illustrates a very rare variety of femoral hernia appendicitis. The appendix was not contained in the hernia sac. Instead, only appendiceal pus was encountered in the hernia sac. Zuckerkandl first described this phenomenon in 1891, albeit within an inguinal hernia sac. ${ }^{6}$ As with any peritoneal recess, the hernia sac may contain pus secondary to any intraperitoneal infectious process. The pus causes distention and inflammation of the sac, which was mistaken for strangulated hernia. The most common underlying pathology is acute appendicitis. The appendix may be either in close proximity to or distant from the hernia sac. There is usually no evidence of intestinal obstruction, which will raise suspicion of strangulated omentum or Richter's hernia.

Once the diagnosis of strangulated femoral hernia is made, most surgeons prefer direct surgical intervention to avoid considerable risk of postoperative complications. Thus, the diagnosis of appendiceal pus-containing femoral hernia is made only during the operation. Despite wide availability of $\mathrm{CT}$, the $\mathrm{CT}$ features of pus-containing femoral hernia is almost never reported in the literature. In our patient, the CT showed an inflammatory fluid-filled mass lateral and inferior to the pubic tubercle, a typical location of acute appendicitis, and no evidence of intestinal obstruction. The differential diagnosis is acute appendicitis complicated with a rare complication of pus-filled femoral hernia or a Richter's hernia. As illustrated in our case, the awareness of the $\mathrm{CT}$ findings facilitated the decision on a treatment strategy. Although drainage of purulent content or appendectomy via hernia sac has been reported in many cases in which diagnosis is not expected, it carries a high rate of infectious complications. Separate laparotomy and groin incision would be very helpful in complete dissection and direct excision of the hernia sac. Thus, it prevents spillage of hernia contents and avoids significant morbidity.

There are some controversies surrounding the method of femoral hernia repair in cases of hernial appendicitis or pus-containing femoral hernia. Infection with the use of mesh repair has been reported. We prefer McVay hernioplasty. However, if no spillage of infectious content occurs, mesh repair is a reasonable alternative.

In conclusion, pus-containing femoral hernia is a rare complication of acute appendicitis. Selective use of CT for evaluating an atypical case of incarcerated femoral hernia may be helpful in early diagnosis and decision on the treatment strategy. The CT features of typical acute appendicitis and incarceration of inflammatory fluid-filled hernia sac without evidence of intestinal obstruction should raise suspicion of this rare entity.

\section{Disclosure}

The authors declare no conflicts of interest in this work.

\section{References}

1. Carry LC. Acute appendicitis occurring in hernias: a report of 10 cases. Surgery. 1967;61:236-238.

2. Akopian G, Alexander M. de Garengeot hernia: appendicitis within a femoral hernia. American Surgeon. 2005;71:526-527.

3. Luchs JS, Halpern D, Katz DS. Amyand's hernia: prospective CT diagnosis. J Comput Assist Tomogr. 2000;24:884-886.

4. Fukukura Y, Chang SD. Acute appendicitis within a femoral hernia: multidetector CT findings. Abdom Imaging. 2005;30:620-622.

5. D’Ambrosio N, Katz D, Hines J. AJR Am J Roentgenol. 2006;186: 906-907.

6. Zuckerkandl M. Hernia inflammata in Folge Typhilitis des Wormfortsatzes in einem Leistebruche. Wiener Klinische Wochenschrift. 1981;4:305. In German.

\section{Publish your work in this journal}

The International Journal of General Medicine is an international, peer-reviewed open-access journal that focuses on general and internal medicine, pathogenesis, epidemiology, diagnosis, monitoring and treatment protocols. The journal is characterized by the rapid reporting of reviews, original research and clinical studies across all disease areas.

\section{Dovepress}

A key focus is the elucidation of disease processes and management protocols resulting in improved outcomes for the patient. The manuscript management system is completely online and includes a very quick and fair peer-review system. Visit http://www.dovepress.com/ testimonials.php to read real quotes from published authors. 